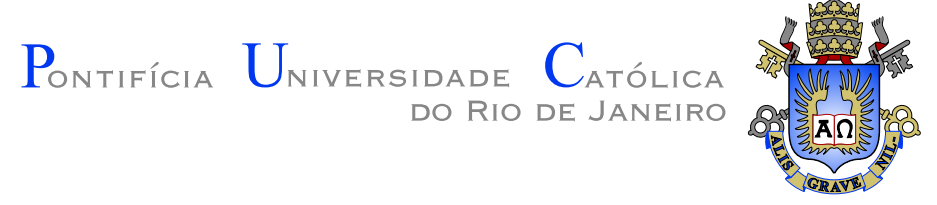

Glaudson Mosqueira Bastos

\title{
Inovação tecnológica: contribuição da metrologia e do usuário gerador de inovação
}

Dissertação apresentada ao Programa de Pós-graduação em Metrologia da PUC-Rio como requisito parcial para obtenção do título de mestre em Metrologia. Área de Concentração: Metrologia para Qualidade e Inovação.

Orientador: Prof. Maurício Nogueira Frota, PhD Co-Orientador: Prof. Antonio José Junqueira Botelho, PhD 


\title{
Glaudson Mosqueira Bastos
}

\section{Inovação tecnológica: contribuição da metrologia e do usuário gerador de inovação}

Dissertação apresentada como requisito parcial para obtenção do grau de Mestre pelo Programa de Pós-graduação em Metrologia do Centro Técnico Científico da PUC-Rio. Aprovada pela Comissão examinadora abaixo assinada.

\author{
Prof. Maurício Nogueira Frota, PhD \\ Orientador \\ Programa de Pós-Graduação em Metrologia \\ PósMQI - PUC-Rio
}

Prof. Antonio José Junqueira Botelho, PhD

Co-Orientador

Programa de Pós-Graduação em Metrologia

PósMQI - PUC-Rio

Prof. Richard Magdalena Stephan, PhD

Programa de Pós-Graduação em Engenharia Elétrica da

UFRJ/Coppe

Prof. Ruth Epsztejn, DSc

CEFET/RJ e Rede de Tecnologia do Rio de Janeiro

Prof. José Eugenio Leal, PhD

Coordenador Setorial de Pós-Graduação do Centro Técnico

Científico - PUC-Rio

Rio de Janeiro, 31 de Julho de 2008 
Todos os direitos reservados. É proibida a reprodução total ou parcial do trabalho sem autorização da universidade, do autor e do orientador.

\section{Glaudson Mosqueira Bastos}

Graduação em Marketing e Comunicação Social (1996). PósGraduação em Marketing pela Universidade Cândido Mendes (1998). MBA em Finanças pelo Instituto Superior de Estudos Pedagógicos (1999). Membro da American Society for Quality, divisão Product Safety and Liability desde 2001. Facilitador da Rede de Cooperação Brasil + e do Movimento Brasil Competitivo (2002-2003). Diretor Adjunto da Regional Rio da Sociedade Brasileira de Metrologia (2004-2005). Associado da ABNT desde 2006. Auditor Interno (NBR ISO 9001:2000) formado pela Det Norske Veritas (DNV), São Paulo, 2006 e pelo Bureau Veritas (BVQI), Rio de Janeiro, 2007. Atualmente é Pesquisador Associado do Núcleo de Estudos e Pesquisas Gênesis em Empreendedorismo, Capital de Risco e Inovação da Pontifícia Universidade Católica do Rio de Janeiro (NEP Gênesis/PUC-Rio) e Gerente de Projetos da LOGIKE ASSOCIADOS S/C, atuando com projetos de desenvolvimento nos segmentos de autopeças, software, metro-ferroviário, naval e de mineração e metalurgia.

Ficha Catalográfica

Bastos, Glaudson Mosqueira

Inovação tecnológica: contribuição da metrologia e do usuário gerador de inovação / Glaudson Mosqueira Bastos; orientador: Maurício Nogueira Frota, PhD; co-orientador: Antonio José Junqueira Botelho, PhD - 2008.

$71 \mathrm{f}$. : il. ; $30 \mathrm{~cm}$

Dissertação (Mestrado em Metrologia) - Pontifícia Universidade Católica do Rio de Janeiro, Rio de Janeiro, 2008.

Inclui bibliografia.

1. Metrologia - Teses. 2. Metrologia. 3. Inovação. 4. Gestão da inovação. 5. Normas técnicas. 6. Regulamentação técnica. 7. Boas práticas da regulamentação. I. Frota, Maurício Nogueira. II. Botelho, Antonio José Junqueira. III. Pontifícia Universidade Católica do Rio de Janeiro. Programa de Pós-Graduação em Metrologia. IV. Título. 







\section{Agradecimentos}

Ao orientador Professor Doutor Maurício Nogueira Frota, orientador e coordenador do Programa de Pós-Graduação em Metrologia da PUC-Rio, pela dedicação e zelo com que nos apoiou desde o processo de admissão ao Pós MQI/PUC-Rio até a etapa final de concepção, desenvolvimento e revisão desta dissertação. Tenho acompanhado pessoalmente a persistência do Professor Maurício Frota em prol do aperfeiçoamento dos profissionais envolvidos com metrologia, normalização e qualidade industrial.

Ao co-orientador Professor Doutor Antonio José Junqueira Botelho, com quem aprendi sobre empreendedorismo tecnológico e os percalços do empreendedorismo em nossa Pátria, sobre a perspectiva econômica inclusa na inovação e a quem agradeço, fundamentalmente, pela confiança e pelos aconselhamentos sempre serenos nos momentos mais críticos na conclusão da dissertação. Agradeço a Deus pela oportunidade de conviver com alguém capaz de reunir em si mesmo, senioridade científica e competência técnica sem perder de vista atributos pessoais como gentileza e amabilidade.

Aos professores da PUC-Rio, em especial, Professor Doutor Rui Pitanga, Professor Doutor Glauter Rocha, Professor Doutor Leonardo Lustosa, Professor Doutor Pimenta Bueno, sempre coerentes e empenhados na excelência do ensino, em nome dos quais agradeço ao corpo de professores, técnicos e colaboradores da PUC-Rio.

Aos professores que participaram da Comissão Examinadora, aceitando avaliar o trabalho. 
Ao amigo Engenheiro Pedro Paulo Almeida Silva, 'expressão viva' dos termos metrológicos e com quem tive o privilégio de conviver na PUC-Rio, na Sociedade Brasileira de Metrologia (SBM) e na elaboração, juntamente com o amigo Engenheiro Ricardo Mesquita, do documento orientativo 'Metrologia e sistemas de informações'.

Ao Gerson Toller, Diretor da Revista Ferroviária, que nos permitiu acesso aos pesquisadores premiados na edição 2007 do Prêmio Amsted Maxion de Tecnologia Ferroviária e o do Prêmio ALSTOM de Tecnologia Metroferroviária, assegurando material de extrema relevância para nossa pesquisa.

Ao amigo Engenheiro Amador Orelo, incansável em suas prototipagens e no sonho de termos no Brasil um verdadeiro projeto de Veículos Leves sobre Trilhos (VLT), em nome de quem agradeço a todos os profissionais do METRÔ RIO CONCESSAO METROVIARIA DO RIO DE JANEIRO S/A que contribuíram com nossa pesquisa, extensivo aos estudiosos no País do conceito de "Light Rail Transport" (LRT).

Ao Engenheiro Felipe César Moreira Ciríaco na área de Engenharia de Vagões da MRS LOGÍSTICA S.A., ao Gustavo Monastério na área de Gestão de Ativos da MRS LOGÍSTICA S.A., ao Dr. Félix e Dra. Tida na Direção da MRS LOGÍSTICA S.A., pela inequívoca contribuição neste trabalho.

Ao Antenor Pazini e a todos na AMÉRICA LATINA S.A. (ALL), e ao Abel Passagnolo Sergio na FERROVIA TEREZA CRISTINA S.A., provas vivas do bem que as concessões fizeram ao nosso País. Muito além dos investimentos na recuperação e manutenção de vagões, locomotivas e vias permanentes está o desenvolvimento de gente competente e inovadora!

Ao amigo sempre fiel, Engenheiro Manoel Mendes, Diretor do CEPEFER - CENTRO DE ESTUDOS E PESQUISAS FERROVIÁRIAS, que, em conjunto com o Engenheiro Aurélio Fernandes me ensinaram a fina arte da gestão de suprimentos na indústria metro-ferroviária e a quem desejo sempre muito sucesso. Que Deus nos permita trabalharmos juntos em novas oportunidades!

Aos amigos de caminhada na trilha da Ciência e Tecnologia e da Gestão Empresarial, Adriano Proença, Roberto Alvarez, Armando Clemente, Evandro Peçanha, Marcus Monteiro, Ricardo Wargas, Dolores Lustosa, Marina 
Brochado, Renato Regazzi, Rogério Regazzi, Antônio Batista, Carina Garcia, Beto, Valério Garcia, Cris Maia, Miriam Zitz, Francisca, Rincón, Alcionei, Renato Garcia, Paulo Clébio, Aristóteles Riani, Roselino, Antonio Carlos Diegues, Paulo Bastos Tigre, Maurício Mendes, Tenório, Vieites, Fernanda e ao Liong Khoe.

Ao amigo e excelente acadêmico Jaime Ticona. "Hagan brillar su luz delante de todos, para que ellos puedan ver las buenas obras de ustedes y alaben al Padre que está en el cielo" (Mateo 5:16 - NVI).

Às secretárias do PósMQI, Marcia e Isabel pelo apoio administrativo e à Eline Girardi pela competente editoração.

Aos amigos que fiz na Sociedade Brasileira de Metrologia (SBM), em especial ao Engenheiro Gilberto Oliveira Gomes (que convive comigo mesmo após eu ter questionado sobre a liga platina-irídio do protótipo internacional do quilograma!) e ao Engenheiro Pedro Paulo Novellino, Secretário Executivo da Sociedade Brasileira de Metrologia (SBM).

Aos meus conselheiros e amigos Rev. Guilhermino Cunha, Rev. Cid Caldas, Rev. Adelino, Rev. Clinton Lenz César, Presbítero Guaraci Sathler, Presbítero Olympio Bezerra, Don Divaldo Gomes, Marcus Raimundo, Oscar Hue, Lino Marujo, Lino Guimarães Marujo, Vilma, Fábio, Mauro Rocha, Cmte. Werner Gripp e Dieter Gripp, juntos no amor que é o vínculo da perfeição.

A Dra. Mariza Bastos, uma pessoa especial, verdadeira batalhadora, que honrou sempre todos os votos e que fez de cuidar, e muito bem, de seus filhos.

A minha amada esposa Fabiola Bueno - vejo em seus olhos a confirmação do amor de Deus para comigo! A ti e ao nosso João, o meu grande amor e o meu obrigado por tanto carinho e dedicação! "Pelo que chamou Abraão o nome daquele lugar: JEOVÁ-JIRÉ; donde se diz até ao dia de hoje: No monte do SENHOR se proverá" (Gênesis 22:14). 


\section{Resumo}

Bastos, Glaudson Mosqueira; Frota, Maurício Nogueira; Botelho, Antonio José Junqueira. Inovação tecnológica: contribuição da metrologia e do usuário gerador de inovação. Rio de Janeiro, 2008. 71p. Dissertação de Mestrado - Programa de Pós Graduação em Metrologia. Pontifícia Universidade Católica do Rio de Janeiro.

A dissertação de mestrado desenvolve-se em sintonia com as prioridades do Programa de Pós-Graduação em Metrologia para Qualidade e Inovação da PUC Rio, inserindo-se na sua linha de pesquisa Negócios, Inovação e Metrologia. Dois são os objetivos do presente trabalho: (i) identificar os mecanismos de contribuição da metrologia ao processo de inovação e (ii) caracterizar o papel do usuário beneficiário da inovação nesse processo. A metodologia empregada compreende uma revisão da literatura especializada sobre os temas da pesquisa e o desenvolvimento de um estudo de caso na indústria metro-ferroviária brasileira. A amostra intencional é constituída por profissionais de $\mathrm{P} \& \mathrm{D}$ de algumas das principais concessionárias brasileiras de transporte ferroviário de cargas e de passageiros. O trabalho se desenvolve no contexto do papel crescente da inovação tecnológica como estratégia para competitividade empresarial e inserção externa das empresas brasileiras, um dos pilares da Política Industrial Tecnológica e de Comércio Exterior (PITCE) e de sua sucedânea Política de Desenvolvimento Produtivo (PDP) lançada em maio de 2008. Em seus resultados, o trabalho analisa, em um primeiro momento, (i) o tipo de inovação promovida na indústria metro-ferroviária e (ii) a conformidade dos projetos inovadores nesta indústria com as normas emanadas do "Comitê Brasileiro de Normalização ABNT/CB-06 - Metroferroviário" da Associação Brasileira de Normas Técnicas (ABNT), para então avaliar (iii) o papel da metrologia e dos usuários no processo da inovação tecnológica propriamente dita. As conclusões destacam que a indústria metro-ferroviária no Brasil adota um viés conservador na seleção de projetos de inovação e que a prática da inovação induzida por usuários ainda não se encontra plenamente difundida, em que pese o elevado nível de acesso dos usuários às especificações de projeto e desenvolvimento de produto. Outrossim, o trabalho permitiu concluir que, no caso da indústria metro-ferroviária, as normas técnicas e regulamentos técnicos impulsionam e orientam o processo de inovação.

\section{Palavras-chave}

Metrologia. Inovação. Gestão da inovação. Normas técnicas. Regulamentação técnica. Boas práticas da regulamentação. 


\section{Abstract}

Bastos, Glaudson Mosqueira; Frota, Maurício Nogueira; Botelho, Antonio José Junqueira. Technological innovation: Contributions of metrology and user-led innovation. Rio de Janeiro, 2008. 71p. MSc. Dissertation - Programa de Pós Graduação em Metrologia. Pontifícia Universidade Católica do Rio de Janeiro.

The subject of the master's dissertation reflects the priorities of the Pontifical Catholic University of Rio de Janeiro's Graduate Program in Metrology for Quality and Innovation, particularly its research line Business, Innovation and Metrology. The research has two objectives: (i) to identify the mechanisms through which metrology contribute to innovation and (ii) to characterize the role of innovationbenefited users in the very innovation process. The methodology employed comprises a literature review of the topic and development of a case study in the Brazilian subway-railway transportation and equipment industry. An intentional sample was formed by R\&D professionals of the major passenger and freight railway Brazilian concessionaire firms. The work is developed in the context of the growing role of innovation as a strategic component of business competitiveness and of innovation as one of the pillars of the country's Foreign Trade and Industrial and Technology Policy (PITCE), and its successor Productive Development Policy (PDP), launched in May 2008. The research project results analyze: (i) the type of innovation encouraged by the subway-railway industry and (ii) the conformity of innovative projects in the industry with norms issued by the "National Standards Committee ABNT/CB-06 - Subway-railway" of the Brazilian Technical Standards Association (ABNT), and then (iii) assesses the role of metrology and of innovation users in the very innovation process. The conclusions highlight that the subway-railway industry adopts a conservative approach in the selection of innovation projects and that the practice of user- induced innovation is not yet fully diffused, in spite of the high level of user access to project and product development specifications. Furthermore, the work allowed us to conclude that, in the case of the subway-railway industry, technical norms and regulations promote and orient the innovation process.

\section{Keywords}

Metrology. Innovation. Management of innovation. Technical standards. Technical regulations. Good practices in regulations. 


\section{Sumário}

1 Introdução $\quad 15$

1.1 A metrologia como "insumo metrológico" na competitividade $\quad 16$

$\begin{array}{lll}1.2 & \text { O respeito ao legado do SINMETRO } & 18\end{array}$

1.3 Inovação e demandas por metrologia 20

$\begin{array}{lll}1.4 & \text { Objetivos do trabalho de pesquisa } & 21\end{array}$

$\begin{array}{ll}1.5 & \text { Preceitos metodológicos } \\ 1.6 & 22\end{array}$

$\begin{array}{lll}1.6 & \text { Estrutura dos capítulos } & 23\end{array}$

2 O sistema de inovação e as demandas por metrologia $\quad \mathbf{2 4}$

2.1 Inovação como componente da produção industrial 24

2.2 Taxonomia básica para se imprimir um significado ao conceito de inovação 27

2.2.1 Alinhamento do conceito de inovação com o Manual de Oslo 30

2.2.2 Dimensionamento do sistema de inovação na perspectiva do Manual de Oslo $\quad 31$

2.2.3 A Inovação como um sistema que precisa ser gerenciado 33

3 Inovação: fontes e inovação conduzida pelo usuário 39

3.1 Movimento unilateral: razões pela customização de produtos 47

3.2 Movimento bilateral: estratégia por 'nichos de inovação' 48

4 Gestão da inovação na indústria metro-ferroviária brasileira e implicações metrológicas

4.1 Pesquisa empírica: a metrologia como elemento estruturante do sistema de inovação presente na indústria metro-ferroviária brasileira $\quad 52$

4.2 Relevância econômica e científica da pesquisa no setor metro-ferroviário 52

4.3 Justificativas quanto ao método 54

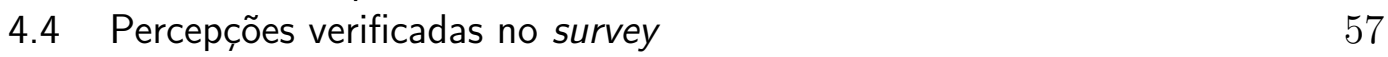

5 Conclusões $\quad 62$

5.1 Mecanismos da contribuição da metrologia 62

5.2 O papel do usuário beneficiário da inovação nesse processo 63

5.3 Recomendações para desdobramentos do trabalho 63

6 Referências bibliográficas $\quad 64$

$\begin{array}{lll}\text { A } & \text { Apêndice A } & 69\end{array}$ 


\section{Lista de figuras}

2.1 Despesa doméstica bruta com P\&D (relação com o PIB) 27

2.2 Teoria 'Chain-link Model' de Kline e Rosemberg. 33

2.3 Estrutura de mensuração da inovação no Manual de Oslo 34

2.4 Modelo Sistêmico de Inovação $\quad 35$

2.5 Fluxo Não Linear de Inovação 36

3.1 Fluxo não linear de ações que demandam a participação do usuário no processo inovativo.

4.1 Fluxo de aplicação do survey 


\section{Lista de tabelas}

1.1 Principais manufaturados brasileiros exportados (janeiro de 2008 versus janeiro de 2007

2.1 Inovação radical pesquisada pela Lally School of Management 30

2.2 Distinção entre Inovação de Produto e de Processo 32

2.3 Processamento de Sinais e socialização da inovação 37

3.1 Elementos e pontos de análise no desenvolvimento da inovação 40

3.2 Estudos sobre freqüência de inovação conduzida pelo usuário 46

3.3 Fonte de inovações de acordo com a natureza das melhorias efetuadas 49

4.1 Fundamentos do Projeto 'Vagão Plástico' 58

4.2 Exemplo de projeto de inovação em tecnologia ferroviária com dimensão de retorno financeiro 


\section{Sumário das notações}

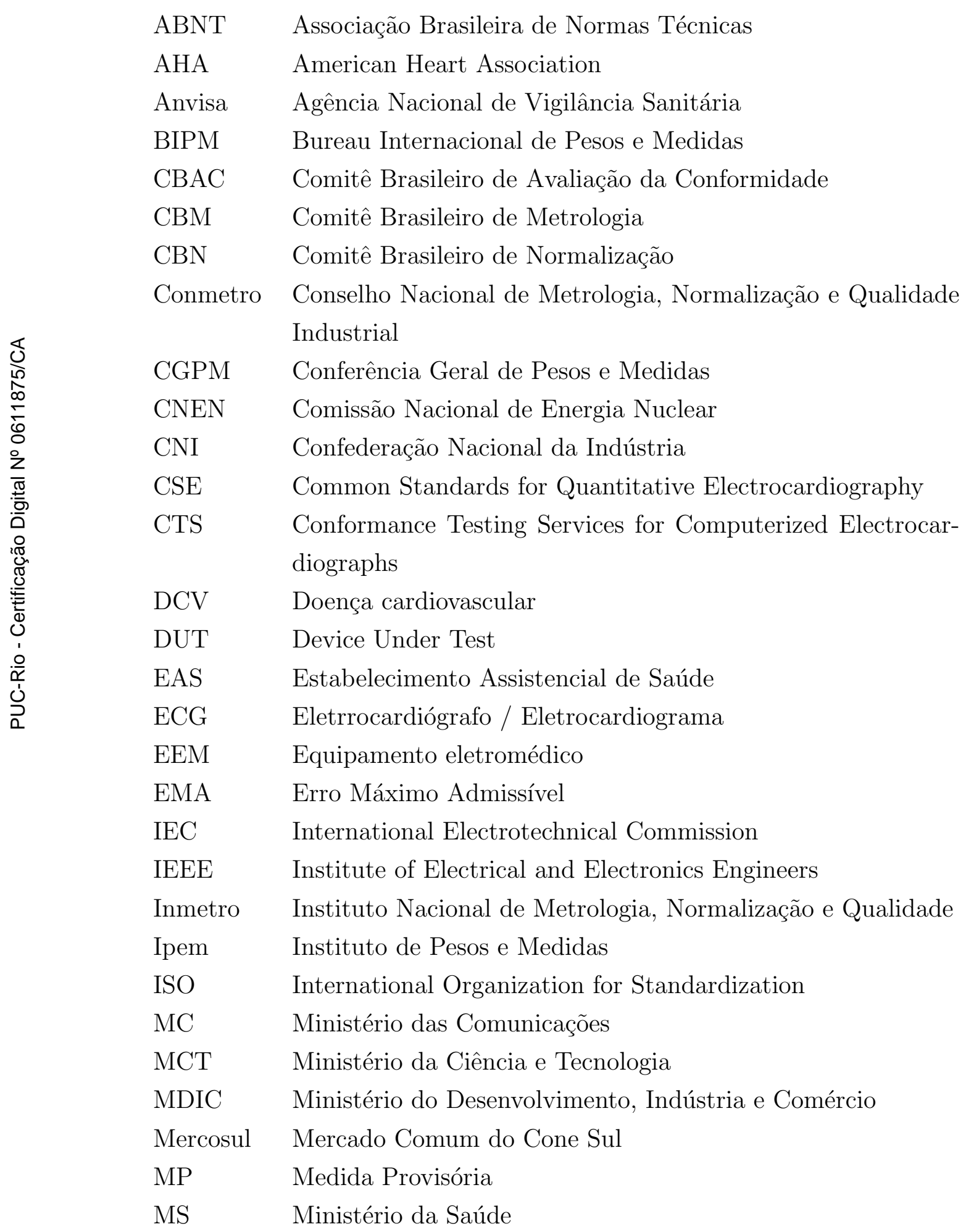


NBR Norma Brasileira Registrada

OIML Organização Internacional de Metrologia Legal

OIN Organismo Internacional de Normalização

OMS Organização Mundial de Saúde

PAME-RJ Parque de Material de Eletrônica da Aeronáutica do Rio de Janeiro

PósMQI Programa de Pós-Graduação em Metrologia para Qualidade e Inovação

PTB Physicalische Technische Bundesanstalt

PUC-Rio Pontifícia Universidade Católica do Rio de Janeiro

RBC Rede Brasileira de Calibração

RBMLQ-I Rede Brasileira de Metrologia Legal e Qualidade - Inmetro

RT Regulamento Técnico

RTM Regulamentação Técnica Metrológica

SABIO Sistema Interativo para Aquisição e Análise de Sinais Biomédicos

SBAC Sistema Brasileiro de Avaliação de Conformidade

SBC Sociedade Brasileira de Cardiologia

SBN Sistema Brasileiro de Normalização

SI Sistema Internacional de Unidades

SIM Sistema de Infrormações sobre Mortalidade

Sinmetro Sistema Nacional de Metrologia, Normalização e Qualidade

VIM Vocabulário Internacional de Termos Fundamentais e Gerais de Metrologia

WHO World Health Organization 